\title{
EVALUACIÓN DE VARIABLES PARA LA PRODUCCIÓN DE METIL ÉSTERES A PARTIR DE ACEITE DE SEMILLA DE ALGODÓN ASISTIDO POR ULTRASONIDO
}

\author{
Dr. Walter G. Morales ${ }^{1}$, Ing. Vanessa Zurkoํ, Ing. Alfredo Sequeira ${ }^{1}$, Dra. \\ Ester Chamorro ${ }^{1}$,
}

Resumen. El uso de la radiación ultrasónica como una nueva herramienta de mezcla, más eficiente en la producción de Biodiesel brinda una alternativa a los métodos convencionales de producción. La Energía del ultrasonido mejora la transferencia de masa entre los reactivos no miscibles a través de la cavitación ultrasónica lo que genera no solo una mayor eficiencia en la reacción y ahorro de tiempo, sino que además puede ser económicamente viable, ya que requiere poca cantidad de catalizador y sólo entre un tercio y la mitad de la energía que se consume por agitación mecánica debido a que maximiza el área superficial de la interfase entre los reactivos no miscibles y con consumos de energía más bajos que la de los reactores agitados. En este trabajo se evalúan los resultados de reacciones asistidas por ultrasonido utilizando un plan de trabajo donde se varían los parámetros tiempo de reacción, relación molar aceite/alcohol, cantidad de catalizador y temperatura; manteniendo constantes los parámetros de frecuenta y potencia del baño de ultrasonido. Se lograron conversiones superiores al 90\% de triglicéridos a metil ésteres.

Palabras Clave: Biodiesel, Ultrasonido; Transesterificación, Metil Ésteres.

\section{INTRODUCCIÓN}

El aumento del consumo de los combustibles fósiles acorta significativamente la vida útil de esta fuente de energía, es por esto que se ha hecho necesario el desarrollo de combustibles alternativos. Los biocombustibles, como el biodiesel, se presentan como una opción al diésel provenientes del petróleo, ya que se obtienen de fuentes de energías limpias y renovables $[1,2]$.

El biodiesel presenta varias ventajas sobre el diésel de petróleo, entre las que pueden mencionarse que es biodegradable, no tóxico, no contribuye a aumentar el efecto invernadero ya que el dióxido de carbono producido durante la combustión se reincorpora al ciclo de carbono, y tiene menos emisiones de hidrocarburos y azufre. Además el número de cetano, la viscosidad, el calor de combustión y la potencia que se obtiene a partir de su uso en motores de ciclo diesel son similares a los del diésel de petróleo $[1,3]$.

La forma más común de sintetizar biodiesel es mediante una reacción de transesterificación, en la cual un triglicérido reacciona con un alcohol de cadena corta, generalmente metanol, en presencia de un catalizador que puede quedar en fase ho-

${ }^{1}$ Centro de Investigación en Química Orgánica Biológica (QUIMOBI) Facultad Regional Resistencia (FRRe), Universidad Tecnológica Nacional (UTN), French, 414, 3500, Resistencia, Chaco, Argentina. 
mogénea o heterogénea. Debido a la naturaleza reversible de esta reacción, es necesario emplear exceso de alcohol para favorecer el equilibrio hacia la formación de biodiesel $[1,2,4]$.

Comúnmente a nivel industrial, la producción de biodiesel se lleva a cabo en reactores discontinuos donde el alcohol, el triglicérido y el catalizador son alimentados al reactor y se someten a constante agitación vigorosa, manteniendo la temperatura de reacción constante que varía entre $50^{\circ} \mathrm{C}$ y $90^{\circ} \mathrm{C}$. El tiempo de reacción requerido para lograr una conversión comercialmente aceptable es de aproximadamente 60 minutos $[1,2,4]$.

La tecnología de producción de biodiesel, constituye uno de los principales obstáculos para la comercialización de éste a gran escala, debido al alto costo y rendimientos variables logrados con el uso de tecnologías tradicionales. Debido a ello, actualmente, la investigación se centra en el desarrollo de métodos tecnológicos alternativos para la producción de biodiesel. Entre estos métodos se encuentra el uso de irradiación con ondas de ultrasonido como una nueva herramienta de mezcla, más eficiente en la producción de biodiesel $[5,6]$.

El uso de ultrasonido en la producción de biodiesel tiene varias ventajas con respecto a la síntesis clásica. Las ondas de ultrasonido mejora la transferencia de masa entre los reactivos no miscibles a través de la cavitación ultrasónica, lo que genera no solo una mayor eficiencia en la reacción y ahorro de tiempo, sino que además puede ser económicamente viable, ya que requiere sólo entre un tercio a la mitad de la energía que se consume por agitación mecánica debido a que maximiza el área superficial de la interfase entre los reactivos no miscibles y con consumos de energía más bajos que la de los reactores agitados convencionales. Los reactores de ultrasonidos pueden ser atractivos en vista a mejorar el rendimiento del proceso de transesterificación mediante la obtención de rendimientos más altos de biodiesel y tiempos de reacción más cortos [5-10].

El propósito de este trabajo fue realizar el estudio del efecto de las variables de proceso de la reacción de transesterificación de aceite de semilla de algodón pre tratado, con catálisis homogénea en reactor batch, en presencia de irradiación ultrasónica, en un baño de ultrasonido, y comparar estos resultados con los hallados en trabajos previos realizados con agitación mecánica [4].

\section{MATERIALES Y MÉTODOS}

\subsection{Materiales}

El metanol (CH3-OH) e hidróxido de sodio $(\mathrm{NaOH})$ fueron comprados de comercios y usados como se recibieron; ambos reactivos de calidad Pro Análisis. El aceite de semilla de algodón fue provisto por la empresa Tante Lote, dedicada a la obtención de éste.

Se utilizó un equipo de Ultrasonido marca Arcano, Modelo PS-30 A con capacidad de 6,5 litros, de 180 watts de potencia, tamaño de unidad de 330x180x310 $\mathrm{mm}$, tamaño del tanque de 300x150x150 $\mathrm{mm}$, voltaje de AC 220-230 voltios/ 50-60 $\mathrm{hz}$, tiempo de trabajo de 1 a 30 minutos y frecuencia de $40 \mathrm{Khz}$.

\subsection{Reacciones de trasesterificación}

Las reacciones de transesterificación se llevaron a cabo por duplicado, siguiendo un plan de reacciones que considera como 
variables el tiempo, la relación molar aceite/alcohol, la cantidad de catalizador y la temperatura, a cada una de las variables se le asignaron cuatro niveles, excepto para la temperatura donde se adoptaron tres niveles.

El equipo de reacción fue montado con un balón de 2 bocas de $500 \mathrm{ml}$ conectado a un refrigerante vertical y un termómetro. El balón fue sumergido en un baño agua termostatizado, dentro del baño de ultrasonido, Figura 1.

Una vez finalizada la reacción, el balón se colocó en agua fría para evitar que los reactivos siguieran reaccionando, respetando el tiempo de reacción estipulado por el plan de reacciones. La mezcla de productos se colocó en una ampolla de decantación para separar las dos fases de productos, metil ésteres en fase superior y glicerol en fase inferior. Una vez separadas ambas fases se procedió a un primer lavado de los metil ésteres con una solución de $\mathrm{HCl}$ al 10\% para neutralizar restos de catalizador. Luego del lavado ácido se separaron las dos fases obtenidas, con la fase sobrenadante que contiene los ésteres metílicos se procedió a realizar un segundo y tercer lavado con solución saturada de $\mathrm{NaCl}$.

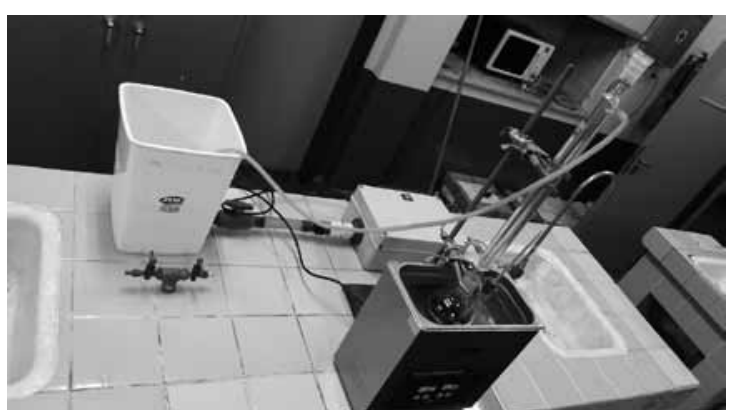

Figura 1: Equipo de reacción en baño de ultraso nido.

Finalizados los procesos de lavados se realizó un centrifugado de los productos obtenidos durante 5 minutos para separar correctamente los metil ésteres de la fase acuosa, finalmente se secó con sulfato de sodio anhidro, se rotularon y guardaron a baja temperatura para su evaluación por cromatografía en placa y gaseosa.

\subsection{Análisis por cromatografía en placa y cromatografía gaseosa}

Los productos obtenidos en los ensayos exploratorios fueron analizado por cromatografía en placa delgada (TLC) usando sílica gel recubierto sobre aluminio, la capa delgada de $0,25 \mathrm{~mm}$ y diámetro de poro medio de 60 micrones, la fase móvil utilizada fue n-hexano-éter etílico en las proporciones 90:10. Se utilizó MnO4K2 como agente cromógeno para visualizar los productos sembrados.

El rendimiento de metil ésteres de cada una de las reacciones fue seguido por cromatografía gaseosa. Se utilizó un cromatógrafo de gases Shimadzu GC 14B, con una columna de tipo Mega Bore DB-1 $(0,53 \mathrm{~mm}$ ID x $1,5 \mu \mathrm{m}$ espesor de film) $\mathrm{Ca}-$ talogo P/N 125-1032 de 2 metros de longitud. Se utilizó nitrógeno como gas carrier fijando la presión de trabajo del mismo en 25 Kpa y la siguiente programación de temperatura: $100^{\circ} \mathrm{C}$, durante 1 minuto, con una rampa de temperatura de $10^{\circ} \mathrm{C}$ por minuto hasta alcanzar los $320^{\circ} \mathrm{C}$ manteniéndose esta temperatura final durante 10 minutos, el detector utilizado fue un FID cuya temperatura se fijo en $320^{\circ} \mathrm{C}$ y la muestra se inyecto manualmente en un volumen de 1 $\mu \mathrm{l}$ en un inyector cuya temperatura se fijó en $320^{\circ} \mathrm{C}$.

\section{RESULTADOS Y DISCUSIONES}

En un reactor discontinuo sumergido en un baño termostatizado de un equipo de ultrasonido, de potencia y frecuencia fijas, se 
llevaron a cabo las reacciones utilizando las siguientes variables: tiempos de reacción: 5 , 10,20 y 40 minutos, inferiores al optimizado en las reacciones con agitación mecánica; relación molar aceite/alcohol: $1 / 3,1 / 6,1 / 7$, y $1 / 9$; cantidad de catalizador: $0,50 \%, 1,0 \%$, $1,5 \%$ y $2,0 \%$ correspondiente a la masa de $\mathrm{NaOH}$ respecto de la masa de aceite; $y$ temperaturas: $25^{\circ} \mathrm{C}, 36{ }^{\circ} \mathrm{C}$ y $60{ }^{\circ} \mathrm{C}$, en este caso también inferiores a las optimizadas en las reacciones con agitación mecánica.

El plan de reacciones utilizado consistió en la variación de un parámetro a la vez, manteniendo todos los demás constantes, tal como puede observarse en la Tabla 1 la potencia del baño de ultrasonido se mantuvo constante durante todos los ensayos en un valor de 180 Watt de potencia y $40 \mathrm{Khz}$ de frecuencia.

Una vez realizadas las reacciones correspondientes al plan de ensayos, la cuantificación de los resultados se llevo a cabo mediante cromatografia en placa y gaseosa donde se obtuvieron los resultados de rendimiento de la reacción de trigliceridos a metil esteres, los cuales se exponen en el Tabla 1.

\begin{tabular}{|c|c|c|c|c|c}
\hline \multirow{2}{*}{ Tiempo } & \multicolumn{2}{|c|}{ Relación molar } & $\begin{array}{c}\text { Cantidad de } \\
\text { catalizador }\end{array}$ & Temperatura & Rendimiento \\
\hline$[\mathrm{min}]$ & Aceite & Alcohol & {$[\%]$} & {$\left[{ }^{\circ} \mathrm{C}\right]$} & $\%$ M.E. \\
\hline 5 & 1 & 7 & 0,5 & 60 & 15,17 \\
\hline 10 & 1 & 7 & 0,5 & 60 & 23,97 \\
\hline 20 & 1 & 7 & 0,5 & 60 & 27,15 \\
\hline 40 & 1 & 7 & 0,5 & 60 & 64,74 \\
\hline 40 & 1 & 3 & 0,5 & 60 & 27,76 \\
\hline 40 & 1 & 6 & 0,5 & 60 & 48,21 \\
\hline 40 & 1 & 9 & 0,5 & 60 & 72,34 \\
\hline 40 & 1 & 7 & 1,0 & 60 & 73,67 \\
\hline 40 & 1 & 7 & 1,5 & 60 & 75,12 \\
\hline 40 & 1 & 7 & 2,0 & 60 & 90,41 \\
\hline 40 & 1 & 7 & 0,5 & 25 & 16,60 \\
\hline 40 & 1 & 7 & 0,5 & 36 & 30,67 \\
\hline
\end{tabular}

Tabla 1: Plan de reacciones y porcentajes de metil ésteres y triglicéridos obtenidos en cada caso.

La Tabla 1 muestra el efecto del tiempo en la reacción de transesterificación de aceite de semilla de algodón, donde se denota que el máximo rendimiento a metil ésteres es del $64,74 \%$ a un tiempo de 40 min. Se pudo observar que a los 5 minutos se obtiene $15,17 \%$ del producto deseado, luego a los 10 y $20 \mathrm{mi}^{-}$ nutos, 23,97 y $27,15 \%$ de metil esteres respectivamente. Con esto, se deduce que a medida que se incrementa el tiempo de reacción se consiguen mayores conversiones, para el caso de estudio con la potencia y frecuencia utilizada.

Una de las variables más importantes que afectan el rendimiento de la reacción es la relación molar aceitelalcohol. En la Tabla 1 se muestra el rendimiento de metil ésteres en función de la variable: relación molar aceite/alcohol, y se observa que con una relación molar aceite/alcohol de $1 / 9$ se obtiene el mayor rendimiento de la reacción, de 72,34\%. 
Esto se explica debido a que con un aumento en la relación molar metanol/aceite, aumenta la cantidad de metanol en la mezcla de reacción, lo que afecta principalmente la intensidad de la cavitación. Un exceso de metanol promueve eventos adicionales de cavitación en el reactor, lo que lleva a la formación de una mejor emulsión, que proporciona espacios adicionales para la reacción y, por lo tanto, una conversión mayor $[6,11]$.

Para estudiar el efecto de la cantidad de catalizador presentes, se varió este de 0,5$2,0 \%$ (en peso de aceite) y se evaluó el rendimiento. Los resultados de este estudio se muestran en la Tabla 1. Se puede observar que un aumento de la concentración del catalizador desde $1 \%$ hasta $2 \%$ origina un aumento significativo de la conversión, logrando el máximo rendimiento con $2 \%$ de catalizador.

Aunque la temperatura habitual de la reacción de transesterificación es la correspondiente al punto de ebullición del alcohol que se utiliza, en este caso metanol, no existen inconvenientes para llevar a cabo este proceso a otras temperaturas.

El efecto de la temperatura en la transesterificación de aceite de semilla de algodón se estudió a tres temperaturas diferentes de $25^{\circ} \mathrm{C}, 36{ }^{\circ} \mathrm{C}$ y $60{ }^{\circ} \mathrm{C}$ como se muestra en la Tabla 1. Se observó que el aumento de la temperatura tiene una influencia favorable sobre la reacción de transesterificación, bajo las condiciones estudiadas. A $60{ }^{\circ} \mathrm{C}$, la reacción obtuvo su mayor rendimiento.

Finalmente, de los resultados presenta- dos puede observarse que se llega a un rendimiento en metiles éstres superior al 90\%. Hay que destacar que, mas allá que la potencia utilizada fue una sola, ésta es utilizada en un equipo de 6,5 litros de capacidad (conteniendo agua, en nuestro caso), dentro del cual se coloca el balón de reacción, donde se encuentra tan solo $70 \mathrm{ml}$ en total de mezcla de reacción, por lo tanto la potencia utilizada no se aprovecha completamente en la mezcla de reacción, sino en los 6,5 litros de baño.

\section{CONCLUSIONES}

En este estudio se pudo observar que en la reacción de transesterificación de aceite de semilla de algodón y metanol con $\mathrm{Na}(\mathrm{OH})$ como catalizador, en presencia de irraciación de ultrasonido sin agitación mecánica, los resultados fueron aceptables y prometedores, ya que se observaron conversiones de hasta el 90\% de los trigliceridos a metil ésteres, trabajando con tiempos (máx. 40minutos) y temperaturas $\left(60^{\circ} \mathrm{C}\right)$ inferiores a las optimizadas con agitación mecánica convencional.

La máxima conversión se observo con relación molar aceite/alcohol 1/9; cantidad de catalizador 2\%; temperatura de reacción de $60^{\circ} \mathrm{C}$ y tiempo de 40 minutos, en un equipo baño de ultrasonido de $40 \mathrm{kHz}$ de frecuencia y $150 \mathrm{~W}$ de potencia.

Comparando este trabajo con los realizados y presentados previamente con agitación mecánica, se observan muy buenos rendimientos pero aún será necesario trabajar sobre el diseño del equipo de ultrasonido utilizado.

\section{Referencias}

1. Fangrui Ma, Milford A. Hanna, "Biodiesel production: a review", Bioresource Technology, vol. 70, p. $1-15,1999$ 
2. Jon Van Gerpen, “Biodiesel processing and production”, Fuel Processing Technology, vol. 86 p. 1097 1107,2005

3. W.G Morales, N. L Polich, A. F Sequeira, E. R Chamorro, E. Herrero, «Biodiesel de aceite de semilla de algodón. Propiedades y formación de precipitados a bajas temperaturas. Performance en motores diesel». Universidad Tecnológica Nacional en el Nordeste Argentino. Investigación y Desarrollo en la Facultad Regional Resistencia, ISBN: 978-987-27897-6-3. Vol. 1, p. 45-51, 2012.

4. W.G Morales, E. P. Dagnino, A. Díaz, A. Sequeira, E. R Chamorro, E. Herrero, «Transesterificación de triglicéridos de algodón catalizada por álcalis». AVERMA. vol. 13(6), p. 87-92, 2009.

5. V. B. Veljkovic, J. M. Avramovic, Olivera S. Stamenkovic , «Biodiesel production by ultrasound-assisted transesterification: State of the art and the perspectives», Renewable and Sustainable Energy Reviews, vol. 16, p. 1193-1209, 2012.

6. A.C.D Vicente Pardal, «Obtención de Biodiesel por transesterificación de aceites vegetales: nuevos métodos de síntesis,» Tesis Doctoral, Universidad de Extremadura-Facultad de Ciencias-Departamento de Ingeniería Química y Química Física, p. 1-323, 2012.

7. D.V Manha, Y.H Chen, C.C Chang, C.Y Chang, H.D Hanh, N.H Chau, T.V Tuyen, P.QLong, C.V Minh, «Effects of blending composition of tung oil and ultrasonic irradiation intensity on the biodiesel production,» Energy, vol. 48, p. 519-524, 2012.

8. L.T Thanh, K. Okitsu, Y. Sadanaga, N. Takenaka, Y. Maeda, H. Bandow, «Ultrasound-assisted production of biodiesel fuel from vegetable oils in a small scale circulation process,» Bioresource Technology, vol. 101, p. 639-645, 2010.

9. F. F.P. Santos, S. Rodrigues, F. A.N. Fernandes, «Optimization of the production of biodiesel from soybean oil by ultrasound assisted methanolysis» Fuel Process.Technol., vol. 90, p. 312-316, 2009.

10. J. Ji, J. Wang, Y. Li, Y. Yu, Z.Xu, «Preparation of biodiesel with the help of ultrasonic and hydrodynamic cavitation,» Ultrasonics, vol. 44, p. 411-4, 2006.

11. V.G. Deshmane, Y.G. Adewuyi, «Synthesis and kinetics of biodiesel formation via calcium methoxide base catalyzed transesterification reaction in the absence and presence of ultrasound,» Fuel, vol. 107, p. 482-474, 2013. 